\title{
An investigation of the association of thromboembolic disease in patients admitted with COVID-19 in Gloucestershire Royal Hospital
}

\author{
Authors: Seema Alaee, ${ }^{\mathrm{A}}$ Hina Iftikhar, ${ }^{\mathrm{A}}$ Rachel Kaminski, ${ }^{\mathrm{A}}$ Charles Sharp ${ }^{\mathrm{A}}$ and Jessica Bennett ${ }^{\mathrm{A}}$
}

\section{Background}

COVID-19 has been associated with poor outcomes secondary to increased pro-thrombotic state causing venous thromboembolisms (VTE).

The hyperinflammatory process leading to a cytokine storm results in endothelial dysfunction and associated hypoxaemia thought to be responsible for the thromboembolic sequaelae. ${ }^{1}$ This can occur despite VTE prophylaxis. Early use of anticoagulation reduces mortality rate as evidenced by case studies. ${ }^{1}$ Measuring coagulation markers, such as D-dimer, has proven to be useful to detect hypercoagulability in patients with COVID-19. This, together with a computed tomography pulmonary angiography (CTPA), may increase the pick-up rate of VTE.

Where it is not possible to perform a CTPA, empiric anticoagulation has been advocated in certain scenarios, including intubated patients who suddenly desaturate, patients with clinical signs of thromboembolic disease and patients who develop hypoxic respiratory failure with raised D-dimers. ${ }^{2}$

\section{Method}

We performed a prospective audit on patients who were admitted with COVID-19 and treated with either continuous positive airway pressure (CPAP), mechanical ventilation or both. All patients received VTE prophylaxis. We compared the association of D-dimer with radiographic evidence of pulmonary emboli on a CTPA.

\section{Results}

Eighty out of 130 patients had a D-dimer level measured. Of those, 10 had positive CTPAs showing pulmonary emboli and 20 patients had negative CTPAs. D-dimer results ranged from $297-52,186 \mu \mathrm{g} / \mathrm{mL}$.

\section{Discussion}

Raised D-dimer levels can reflect a prothrombotic state, however, do not necessarily correlate with radiographic evidence of pulmonary emboli. A negative CTPA may not account for the profound microembolic burden contributing to hypoxaemic respiratory failure. In particular, rising D-dimer in conjunction with increasing oxygen requirements may be predictors of pulmonary emboli and aid in delivery of prompt treatment.

There may be an association with other comorbidities and the development of pulmonary emboli. Patients with a high BMI are more at risk of developing pulmonary emboli and it is imperative to consider such risk factors when considering anticoagulation. ${ }^{3}$ Providing there are no contraindications, empiric anticoagulation should be continued for at least 3 months.

\section{Conclusion}

Early anticoagulation can help improve mortality rates in these patients and should be considered in patients with high D-dimers, even if the CTPA does not prove pulmonary emboli.

\section{Conflicts of interest}

None declared.

\section{References}

1 American Society of Haematology. COVID-19 and pulmonary embolism. ASH, 2020. www.hematology.org/covid-19/covid19-and-pulmonary-embolism [Accessed 01 October 2020].

2 Abernethy K, Sivakumar P, Patrick T et al. Coexistent COVID-19 pneumonia and pulmonary embolism: challenges in identifying dual pathology. Thorax 2020;75:812-4.

3 Science Daily. Pulmonary embolism and COVID-19. Science Daily, 2020. www.sciencedaily.com/releases/2020/06/200602183408.htm [Accessed 15 September 2020]. 